\title{
Información y ciudadanía, una propuesta desde la gobernanza
}

Héctor Alejandro Ramos Chávez *

Artículo recibido:

16 de mayo de 2014.

Artículo aceptado:

19 de mayo de 2014.

\section{Resumen}

El surgimiento y reconfiguración de una sociedad que cuenta con mayores medios para allegarse de información, la cual tiene nuevas características así como contradicciones, genera la necesidad de debatir en torno al papel de la propia información en la construcción de una ciudadanía más activa y participativa en la gestión de políticas públicas. Tomando en consideración el contexto latinoamericano, este artículo aborda esa discusión vinculándola con otros temas como la democracia, la participación ciudadana, la esfera pública, la era de la información y la sociedad del conocimiento. De igual forma, se reflexiona sobre otros enfoques de gobierno, como el de la gobernanza, que pueden

* Instituto de Investigaciones Bibliotecológicas y de la Información de la UNAM. aramos@iibi.unam.mx

INVESTIGACIÓN BiBLIOTECOLÓGICA, Vol. 29, Núm.67, septiembre/diciembre, 2015, México, ISSN: 0187-358X. pp. 113-140 
permitir que esa participación sea más amplia y activa. Las conclusiones apuntan a que si bien en la región actualmente existen desigualdades en cuanto a la disponibilidad y acceso a la información, la gobernanza ofrece elementos promisorios para la construcción de una ciudadanía más informada y participativa en los asuntos públicos y en la gestión de políticas públicas.

Palabras clave: Información; Ciudadanía; Esfera pública; Políticas públicas; Gobernanza.

\section{Abstract}

Information and citizenship: a governance perspective Héctor Alejandro Ramos-Chávez

The emergence and reconfiguration of a society that has greater means to access information - which in itself presents new features and contradictions - generates a need to debate the role of information in encouraging a more active and participatory citizenship in the management of public policies. This article discusses issues of democracy, citizenship participation, the public sphere, and how these are related to the information age and knowledge society in Latin America. Similarly, it reflects on other aspects of government, such as governance, which can enable wider and more active citizen participation. The article concludes that while inequality exists in the region in terms of availability and access to information, governance offers promising elements to build a more informed and participatory citizenship in public affairs and public policy management.

Keywords: Information; Citizenship; Public Sphere; Public Policies; Governance.

\section{INTRODUCCIÓN}
A pesar de los grandes avances en cuanto a la disponibilidad de informa- ción mediante las nuevas tecnologías de información y comunicación, el acceso a Internet, las bibliotecas públicas y la amplia oferta de literatura 
especializada en prácticamente todos los campos del conocimiento, persiste aún un amplio sector de la sociedad que se encuentra excluido de estos logros. Las implicaciones de este desigual acceso a la información dan lugar al surgimiento de una ciudadanía diferenciada, en la que podemos encontrar a ciudadanos bien informados que cuentan con elementos para participar en los asuntos públicos y a otros que no lo están, lo que ocasiona que su participación sea escasa o nula.

Este sector excluido tiende a formar su opinión a partir de la información dada por los medios masivos de comunicación, principalmente la televisión, lo que trae a colación la temática de la calidad de la información que es "consumida”. Algunos autores advierten de la posibilidad de que los medios de comunicación, en su búsqueda de legitimación, sometan a los ciudadanos y a sus necesidades a un proceso de transformación, lo que da como resultado una ciudadanía estereotipada (Giglia y Winocur, 1996; Martín-Barbero, 2001; Winocur, 2002, 2003).

La solución a estas problemáticas no es sencilla de plantear, pues en gran medida el problema se vincula con los altos índices de desigualdad imperantes en México y América Latina en general, así como con la persistencia de una esfera pública que no incluye a gran parte de la población (Fleury, 2004).

Ante este escenario, surge la necesidad de ahondar sobre la reflexión del papel que juega y la importancia que tiene la información en la conformación de la ciudadanía, así como en la posibilidad de plantear nuevos modelos de gestión, como el de la gobernanza, que permitan incluir a un mayor número de individuos en los procesos de elaboración de las políticas públicas y programas relacionados con la información.

En este sentido, tomando en consideración el contexto de Latinoamérica con algunas anotaciones al caso específico de México, en este trabajo se plantea avanzar en el debate de la importancia que tiene la información en la construcción de ciudadanía y en cómo una ciudadanía más informada puede contar con mejores elementos para participar en la gestión de las políticas públicas. Para ello, en una primera parte del documento se aborda la discusión del concepto de ciudadanía y se brindan algunas nociones generales, así como las tendencias y el debate actual de la noción. La segunda parte analiza el vínculo que existe entre ciudadanía y democracia, y discute la importancia y el papel que juega la información en la construcción de ciudadanía. En el tercer apartado se aborda el concepto de participación ciudadana, contextualizándola en la llamada era de la información y de la sociedad del conocimiento. La cuarta parte del documento analiza el modelo de gobernanza como una forma alternativa en la gestión de políticas públicas, remitiendo la discusión en un primer momento a conceptualizar el término y diferenciarlo 
del de gobernabilidad. Por último se dan algunas conclusiones y se proponen algunos derroteros de investigaciones futuras.

\section{LA DISCUSIÓN SOBRE LA CIUDADANÍA}

Aunque no es objetivo de este trabajo realizar un minucioso desarrollo del concepto de ciudadanía, es pertinente establecer un marco referencial para entender el uso que se le da al concepto. La noción de ciudadanía ha tenido distintos enfoques según el punto central de análisis del que se parta, entre otros estudios se la ha vinculado con una forma de control social (Vieira, 1998), con su relación con la ciudad y los espacios públicos (Borja, 1998; Ramírez, 2003; Capron y Monnet, 2003; Espinosa, 2004), con la interacción que establece con el Estado mediante su capacidad de generar mayores niveles de democracia (O’Donnell, 1993), con relación al punto de vista de género (Bolos, 2008; Molyneux, 2000; Aguirre, 2003), con la manera en que se juegan las circunstancias de desigualdad social (Fleury, 2004), así como con las nuevas formas que la ciudadanía ha adquirido en función de los fenómenos de migración y globalización mundial (Lechner, 2000).

De igual forma, dependiendo de los contextos culturales e históricos en los cuales se inserte el análisis, la ciudadanía ha asumido diferentes formas e interpretaciones, la más común de ellas la de vincularla con su forma más básica, definiéndola como el derecho a tener derechos (Vieira, 1998). Sin embargo, la noción más amplia y reconocida, tanto en su significado como en sus elementos constitutivos, se atribuye a T. H. Marshall (1997) en su análisis del desarrollo del concepto de ciudadanía para el caso de Inglaterra. ${ }^{1}$ En este sentido, Marshall propone que la ciudadanía está compuesta por tres elementos:

1. El elemento civil, referente a los derechos que son necesarios para la libertad individual, como la libertad de la persona, de expresión, de pensamiento y de religión, así como por el componente del derecho a la propiedad y a la justicia.

2. El elemento político, que se relaciona con el derecho a participar en el ejercicio del poder político.

3. El elemento social, que se vincula con el derecho a tener un mínimo bienestar económico, así como con el derecho a ser parte del patrimonio social y a contar con estándares civilizados de vida. 
Se le han hecho algunas críticas a esta división de los componentes que conforman la ciudadanía. Craston (1983) menciona que no se pueden entender los derechos sociales como derechos naturales, y por ende darles carácter de principios universales, sino que éstos surgen basados en los procesos históricos de cada nación, por lo que no se podrían vincular con una concepción general de ciudadanía. Por otra parte, Turner (1990) critica la visión de que el ciudadano aparece como un actor pasivo supeditado a las decisiones y acciones de las instituciones del Estado. Finalmente, Roche (1987) sostiene que los aportes de Marshall no reconocen los procesos de acción política, como a su parecer fueron las revoluciones, que dieron pie a la construcción y el entendimiento del concepto de ciudadanía.

A pesar de las críticas, los aportes de Marshall permitieron ahondar en el debate de la vinculación de la ciudadanía con el de clase social, que según este mismo autor parten de principios contradictorios, pues la ciudadanía se define con base en el principio de igualdad de derechos y obligaciones, mientras que la clase social se estructura como un sistema de desigualdades. Ante este escenario, la ciudadanía tiene el reto de no quedarse en el reconocimiento formal de una capacidad igual para disfrutar los derechos, sino de establecerse como un principio real de igualdad que conlleve una justicia social. Para ello, resulta necesario encontrar un equilibrio entre lo civil, entendido como los derechos individuales, y lo cívico, relacionado con los deberes de los individuos con el Estado.

Hasta aquí se podría identificar una visión "histórica" del concepto de ciudadanía en la cual el individuo es nominado como tal, y por lo cual cuenta con derechos jurídicamente establecidos tanto por las leyes jurisdiccionales de las naciones como por las normas de carácter universal que refleja desde 1948 la Declaración Universal de los Derechos Humanos. Sin embargo, a los elementos primarios constitutivos de la ciudadanía se le agregan, a partir de las discusiones que se dan en la segunda mitad del siglo XX, los derechos que no recaen en la persona, sino en colectividades humanas como la comunidad, la nación o grupos étnicos; así como en derechos como el del medio ambiente, de las mujeres, ancianos, niños, jóvenes y consumidores. Incluso, se ha hablado de derechos, bajo la etiqueta de "cuarta generación", relativos a la bioética para preservar la vida y a la regulación de la ingeniería genética (Vieira, 1998).

En este sentido han surgido diversas vertientes que han abordado enfoques que se deben tener en consideración para comprender el sentido del concepto de ciudadanía en la actualidad. Algunos de estos enfoques se relacionan con el surgimiento de nuevas formas de identificación social, problemas de representación política tradicional, búsqueda de nuevos canales 
de participación, conflictos étnicos y el fenómeno de la globalización, entre otros. Todos estos elementos impactan los significados tradicionales del concepto de ciudadanía (Winocur, 2003).

Para contextualizar el sentido del concepto de ciudadanía en la actualidad, se considera pertinente abordar, de forma sucinta, tres de estos enfoques. El primero está relacionado con la pérdida de credibilidad y representatividad de los canales tradicionales de participación como son los partidos políticos o sindicatos; el segundo tiene que ver con el surgimiento de nuevas identidades y su lucha por su visualización y reconocimiento; y el tercero está relacionado con la influencia que ejercen los medios de comunicación en la construcción de la ciudadanía.

Con relación al primer enfoque se ha argumentado que "la política ya no es lo que fue. Ella fue un conjunto de instituciones y procedimientos más o menos claramente estructurados, con amplia penetración en la sociedad a través de mecanismos clientelares y corporativos [...] hoy en día la situación ha cambiado" (Lechner, 2000: 25). En este sentido, se da cuenta de la pérdida de centralidad de la política como eje principal que durante muchos años pudo ordenar y articular la vida social, donde los ciudadanos fincaban sus expectativas y utilizaban sus canales para que se cumpliera ese ordenamiento social. Sin embargo, han existido cambios en el sistema funcional de los ámbitos económico, social y político, que han cuestionado el mantenimiento del orden social proporcionado anteriormente por la política.

Este descrédito de los canales tradicionales de participación también se relaciona con una situación de crisis en cuanto a las ideologías y representaciones de los partidos políticos y de los sindicatos, lo que impacta en que "el ejercicio de la ciudadanía se desplaza de la política institucional a diversas instancias y núcleos de organizaciones sociales, locales y comunitarias con intereses diversificados" (Winocur, 2003: 234).

Junto con este enfoque, y quizá como una respuesta a él, está el surgimiento de nuevos grupos con identidades diferenciadas, y la necesidad de éstos de tener un reconocimiento, así como el cumplimiento de sus demandas y necesidades específicas. Aunque estos movimientos acerca de nuevas identidades surgieron con mayor fuerza en otras áreas geográficas como Europa y los Estados Unidos de América, su importancia ha ido en aumento en los países de la región latinoamericana. Estas discusiones han arrojado un debate muy importante en cuanto al sentido del significado del ciudadano, dejando de lado el reconocimiento de igualdad por derechos al ser ciudadano, al pasar a ser reconocidos y tener derecho a ser diferentes.

Como grupos que incluyen a este sector se encuentran los grupos de feministas que abordan el debate de las necesidades específicas de la mujer; 
pero de igual forma se hace necesario conocer los intereses, derechos específicos y formas de participación de los indígenas, migrantes, adultos mayores, discapacitados, jóvenes y las minorías raciales y sexuales. Muchos de estos grupos no se sienten plenamente identificados con el carácter general de ciudadanía y buscan, a partir de su propia diferencia, ser tomados en consideración como integrantes de la sociedad. En otras palabras, estos grupos demandan su reconocimiento y que se

acoja su modo de estar en el mundo, su manera de relacionarse con los demás y con la naturaleza, y que se expresa en el lenguaje que usan, en las doctrinas comprehensivas que a veces profesan y en la manera en que relacionan los géneros [...] lo que ellos parecen desear es participar como grupo, con identidad propia, en la comunidad política. (Peña, 2005: 100)

Es a partir de estas discusiones que el concepto de ciudadanía hace referencia tanto a los derechos individuales de las personas como al vínculo de pertenencia a una comunidad específica, no sólo limitándola a la idea genérica de nacionalidad sino, además de ello, a un grupo más fácilmente identificable tal como mujeres, jóvenes, adultos mayores, discapacitados, etcétera.

Como tercer elemento de análisis está el tema de la relevancia que en la actualidad tiene la vinculación de los medios de comunicación con la ciudadanía. En este punto es importante tomar en consideración la influencia que tienen los medios de comunicación en los sentidos que van adquiriendo tanto la práctica como la representación de la ciudadanía, pues

la retracción de los espacios públicos tradicionales, junto con la omnipresencia de la televisión y de la radio en los hogares, han afectado considerablemente los procesos de formación de opinión, las modalidades de participación, las maneras de pertenecer y las estrategias de inclusión en la esfera de lo público. (Winocur, 2003: 237)

Tema relevante en este análisis es el entendimiento de las nuevas modalidades en las que participa la ciudadanía, modalidades muchas veces mediatizadas por la televisión y la radio, y el peligro de que el desempeño ciudadano se vea más relacionado con prácticas de consumo (García, 1995), que con una verdadera preocupación por atender y participar en temas trascendentales de interés común.

En este punto se puede reconocer a los medios de comunicación como actores muy importantes en cuanto a su capacidad para poner a consideración, discutir y establecer asuntos de interés público, pero igualmente resulta oportuno tomar en cuenta que los mismos medios también pueden ocultar, limitar e inhibir la información sobre ciertos temas de la agenda pública. Lo 
anterior traería a discusión el tema de la posibilidad que tienen los medios privados de generar información objetiva y transparente, pues muchas veces están determinados por sus propios intereses tanto comerciales como corporativos. En este sentido, se ha argumentado que

los fines del modelo comercial de medios -el que más rápidamente se expande en el mundo- sí generan controversia para el espacio público en las democracias, pues los intereses económicos, corporativos, e incluso a veces políticos de este tipo de medios, inciden en lo que presentan como contenidos, lo cual puede afectar su desempeño a la hora de vigilar, informar y debatir. (Guerrero, 2006: 13)

En conclusión, la discusión de los medios de comunicación y los medios tradicionales de participación ciudadana no debería centrarse en la superposición de formas del ejercicio de la propia ciudadanía, sino en formas complementarias que permitan construir nuevas formas y escenarios de participación (Martín-Barbero, 1999).

Con estos elementos se aprecia cómo el concepto de ciudadanía ya no puede circunscribirse con exclusividad al plano jurídico como "derecho de tener derechos", sino que entran a discusión muchos otros factores que afectan a las prácticas y formas en las que se desarrolla la ciudadanía, por lo que el entendimiento del concepto retrotrae al análisis y comprensión de otros elementos que se van sumando al entendimiento de la ciudadanía.

Ahora bien, tanto para Marshall como para otros autores que han abordado el análisis de la ciudadanía, ésta sólo se puede dar de forma plena en un Estado democrático, en el cual los derechos civiles, políticos y sociales estén asegurados para todos los integrantes de la sociedad, quienes tengan además la capacidad de participar en los asuntos públicos. Surge aquí la discusión sobre la relación entre la ciudadanía y la democracia, pues como se mencionó, para que surja la primera es necesario que exista un gobierno democrático comprometido con que la ciudadanía se consolide, mientras que debe existir una ciudadanía independiente y lo suficientemente participativa para que la democracia pueda realizarse. Estos elementos serán analizados en el siguiente apartado, incluyendo el tema de la información como elemento esencial en la construcción de ciudadanía.

\section{DEMOCRACIA, INFORMACIÓN Y CIUDADANÍA}

Las discusiones generadas alrededor de la gobernabilidad democrática y el rediseño institucional se han estancado en los límites de la democracia repre- 
sentativa. $^{2}$ En este sentido, se ha argumentado que para alcanzar niveles de acuerdos sociales amplios en la esfera pública, se torna fundamental la asociación entre la propia democracia representativa con la democracia participativa, ${ }^{3}$ asociación que sea capaz, a su vez, de construir políticas públicas eficaces que atiendan de la mejor forma posible las necesidades de la sociedad.

Ahora bien, si tomamos en consideración la descripción de América Latina como una de las regiones con mayores niveles de desigualdad en el mundo, resulta necesario traer a discusión el fenómeno de exclusión en el que se encuentra un gran número de habitantes de la región. Dicha exclusión puede ser de tipo económico, político, cultural y de participación, pero también, y de cierta forma relacionada con todas las anteriores, de acceso a la información. Algunos autores han abordado la temática de la desigualdad existente en el acceso a la información mediante la utilización de las nuevas tecnologías de la información y comunicación bajo la denominación de "brecha digital"4 (Hoffman, Novak y Schlosser, 2001; Castells, 2001; Servon, 2002; Rodríguez, 2006, entre otros). Todos estos elementos generan un restringido acceso a la esfera pública.

Debido a la importancia que cobra el concepto de esfera pública en su relación con la temática de la ciudadanía y la democracia, conviene aquí ahondar en la reflexión de dicho concepto, que en primera instancia tiene como contraparte la esfera privada en la cual destacan elementos como el hogar y la familia. Por el contrario, la esfera pública se relaciona con lo que puede ser visto y escuchado por todos, es decir: "la esfera pública, al igual que el mundo en común, nos junta y no obstante impide que caigamos uno sobre otro, por decirlo así" (Arendt, 1993: 62). De forma resumida, en la obra de Arendt (1993) se menciona que son principalmente tres las actividades en las que se ha basado la vida de la humanidad en la Tierra: la primera es la "Labor", relacionada con todas aquellas tareas necesarias para el mantenimiento de la vida, como alimentarse, beber, dormir y descansar; en segundo lugar el "Trabajo", en donde mediante la utilización de los materiales que ofrece la naturaleza se "fabrica la interminable variedad de cosas cuya suma total constituye el artificio humano" (Arendt, 1993: 165), y por último está la "Acción”, que le da al individuo el sentido de libertad y distingue a la humanidad del resto

2 También conocida como democracia delegativa, pasiva o ciudadanía de baja intensidad.

3 A diferencia de la idea de democracia representativa en donde se podría hablar más de una población o sociedad civil que adquiere un papel meramente legitimador o de censura, mediante la emisión del voto; a las acciones del gobierno, en la propuesta de democracia participativa, activa o deliberativa, sobresale la utilización del concepto del ciudadano, el cual está informado y participa activamente mediante la argumentación de sus ideas en el espacio público.

4 Este concepto hace referencia a la desigualdad en cuanto a la disponibilidad y uso de tecnologías como la computadora, conexión a Internet y telefonía celular, entre otros. 
de la naturaleza, siendo precisamente en esa "acción" en donde "con palabra y acto nos insertamos en el mundo humano" (Arendt, 1993: 206) y con ello la esfera pública cobra sentido, ya que permite que surjan dos elementos esenciales en el entendimiento de la ciudadanía:

1) la posibilidad de que todos los individuos sean vistos y escuchados,

2) la generación de un espacio común en donde los individuos se muestren mediante la argumentación, el discurso y la acción.

Hay algunos puntos en común entre estos aportes y la teoría de la acción comunicativa de Habermas (1987), en la cual la discusión de la esfera pública parte del entendimiento de las sociedades en dos niveles simultáneos de análisis: el "sistema" y el "mundo de la vida". Con relación al "sistema" el propio Habermas indica que son los campos basados en una razón instrumental y relacionados con la política (Estado) y con la economía que, con la evolución y modernización del capitalismo, terminaron por dominar a manera de "colonización" al "mundo de la vida", relacionado éste último con aspectos vinculados con la cultura, la personalidad y la sociedad, aspectos que se fundamentan en una razón comunicativa. Es en este último punto en donde el lenguaje juega un papel fundamental en la generación de acuerdos entre los individuos mediante la utilización del diálogo. En este sentido, Habermas indica que

los mecanismos sistémicos acaban desplazando las formas de integración social, incluso en aquellos ámbitos en que la coordinación de la acción en términos de consenso no tiene sustitución alguna; es decir, incluso allí donde lo que está en juego es la reproducción simbólica del mundo de la vida. Entonces la mediatización del mundo de la vida adopta la forma de una colonización del mundo de la vida. (Habermas, 1987: 276)

Ante este escenario se propone la necesidad de revertir esa "colonización" del "sistema" al "mundo de la vida", con la utilización del principio del discurso, la participación y del diálogo que propicia la acción comunicativa. Con ello se podría dar un cambio en donde

un sistema autorregulado, según el cual a todo suceso o estado se le puede atribuir un significado en virtud de su posición funcional, es sustituido gradualmente por un modelo estructurado en términos de teoría de la comunicación, conforme al cual los actores orientan sus acciones según sus propias interpretaciones de la situación. (Habermas, 1987: 168) 
De esta forma, para Habermas la esfera pública, relacionada directamente con el "mundo de la vida", es el lugar donde se generan decisiones colectivas y por ende donde se legitima la democracia. Sin embargo, el propio Habermas anticipa ciertas problemáticas en el escenario ideal del proceso democrático, como que únicamente se tomen en consideración las virtudes actuales de los ciudadanos, pues esto tendría que suponer que todos cuentan con información suficiente y una correcta asimilación de ésta para fundamentar sus discursos y opiniones. En este punto y retomando a Marshall, "el derecho a la libertad de palabra tiene poca sustancia real si, debido a la falta de educación, usted no tiene nada que merezca la pena decir y carece de medios para hacerse escuchar en caso de que quiera decir algo" (1997: 316). Lo anterior se vincula directamente con el tema de la información, tanto en su entendimiento como derecho, así como en su papel para volver realidad la libertad de palabra.

En un contexto democrático, se puede entender la información como un elemento esencial mediante el cual los ciudadanos pueden incrementar sus conocimientos y, con ello, su capacidad de acción frente a los asuntos públicos; es decir, la información como un pilar de la construcción de la esfera pública. Sin embargo, en el escenario actual de la región nos encontramos con que los grupos marginados, pobres y vulnerables en muchas ocasiones carecen de información importante y oportuna para tener un mejor modo de vida, y también de información relacionada con sus derechos, opciones de empleo, servicios públicos de salud, vivienda y educación, así como de los programas y políticas públicas. Lo anterior deriva en una nula presencia y participación tanto en los asuntos públicos como en la definición de la agenda, de estrategias y de la utilización de los recursos públicos.

En este sentido, se ha argumentado que "la construcción de la democracia en la región introduce la reivindicación ciudadana de un derecho de quinta generación -más allá de los derechos civiles, políticos, sociales y difusos- que equivale a demandar una gestión deliberativa de las políticas públicas" (Fleury, 2004: 142). En este punto, resulta igualmente necesario instrumentar acciones mediante las cuales los hasta ahora excluidos de información puedan acceder a ella, con lo cual tengan mejores elementos para su participación en la gestión de políticas públicas y en general en su participación en los asuntos públicos.

Se puede entender a la información como un derecho así como un principio básico en los gobiernos democráticos. Como derecho, han existido grandes avances en el reconocimiento a nivel internacional de los derechos de libertad de expresión y acceso a la información, que tienen por objetivo ahondar en los propios principios democráticos, relacionados con la parti- 
cipación, la responsabilidad, la rendición de cuentas y en general con la transparencia. Como principio básico, el acceso a la información se puede entender como un instrumento fundamental para aumentar y mejorar la capacidad de acción de la población, pues forma la base en la que se construye la participación en la esfera pública, y con ello en los procesos democráticos y en la gestión de políticas públicas.

Ahora bien, si resulta fundamental la protección del derecho al acceso a la información, entendido como la posibilidad de allegarse a la misma de forma clara, vasta y oportuna, resulta también indispensable mejorar los flujos de información que se dan entre los distintos actores, como son el gobierno, las asociaciones, las organizaciones de la sociedad civil, los grupos de interés y el sector privado. Ante esto, se torna esencial la creación y el reforzamiento de los mecanismos y las políticas que permitan la comunicación y el intercambio de información entre los actores.

En este punto, han existido recomendaciones de los organismos internacionales encaminados a mejorar la situación en esta materia. El Programa de las Naciones Unidas para el Desarrollo (2003) aconseja centrar los esfuerzos principalmente en cuatro áreas:

- Fortalecer el marco legal que regula y permite la libertad y el pluralismo en la información.

- Apoyar y fortalecer, tanto a nivel local como nacional, las capacidades y el surgimiento de redes, así como un mejor nivel de los medios de comunicación, con objeto de propiciar el intercambio de información plural e independiente.

- Acrecentar el conocimiento de los derechos de acceso a la información oficial y una mejora en los canales de suministro y acceso de información.

- Generar y mejorar los mecanismos de comunicación necesarios para que los menos favorecidos tengan la capacidad de incidir en la formulación de políticas, así como en la práctica de los asuntos locales y nacionales.

Como derecho, el acceso a la información ha quedado de manifiesto en la Declaración Universal de los Derechos Humanos, que en su artículo 19 establece: "Todo individuo tiene derecho a la libertad de opinión y de expresión; este derecho incluye el de no ser molestado a causa de sus opiniones, el de investigar y recibir informaciones y opiniones, y el de difundirlas, sin limitación de fronteras, por cualquier medio de expresión". Por su parte, para el caso nacional, en el artículo 6 de la Constitución Política de los Estados 
Unidos Mexicanos se establece que "La manifestación de las ideas no será objeto de ninguna inquisición judicial o administrativa [...] el derecho a la información será garantizado por el Estado” y continúa: "Toda persona tiene derecho al libre acceso a información plural y oportuna, así como a buscar, recibir y difundir información e ideas de toda índole por cualquier medio de expresión”.

Como se puede apreciar, en ambos artículos se toma en consideración tanto el derecho a la información como a la comunicación y manifestación de las ideas. Por lo tanto, los individuos pueden desempeñar el papel de usuarios, conductores y también el de generadores de información. Por lo anterior se puede entender que el acceso a la información no puede quedar exclusivamente relacionado con los temas de protección de los derechos a la información, sino que de igual forma es necesario promover y proteger el uso de esa información mediante la comunicación, lo cual permitiría la expresión de las ideas y una verdadera participación en los procesos democráticos y los asuntos públicos.

De los elementos antes señalados se desprende la necesidad de una reconstrucción de la esfera pública en un ambiente democrático que permita una participación más amplia de un mayor número de individuos, para lo cual es necesario encontrar nuevas formas de organización, negociación y concertación, así como mejores canales que permitan un acceso más amplio a la información relevante y oportuna. Lo anterior ayudaría a sobrepasar el simple optimismo discursivo que destaca la participación ciudadana como elemento esencial para mejorar la situación social y ayudaría a revertir la poca intervención de los ciudadanos en la gestión de las políticas públicas; escasa intervención que tiene su origen, en gran medida, en la precariedad de los elementos sociales básicos que impiden el correcto ejercicio de los derechos ciudadanos (Canto, 2008). Para ello, resulta importante ahondar en la reflexión de otro elemento vinculado al de ciudadanía, que es el de la participación ciudadana y cómo se inserta ésta en la sociedad del conocimiento, mismos elementos que se analizarán en el siguiente apartado del documento.

\section{LA PARTICIPACIÓN CIUDADANA EN LA SOCIEDAD DEL CONOCIMIENTO}

Existen diferencias entre el entendimiento del ciudadano que es nominado como tal de forma prerrogativa, el cual se hace sujeto receptor de derechos, y aquel ciudadano que se informa, interviene y participa en los asuntos públicos de su comunidad. En este punto, surge la necesidad de ahondar en la reflexión del concepto de participación ciudadana y, debido a las transforma- 
ciones en materia de tecnologías de información y comunicación, de contextualizarla en la era de la información y sociedad del conocimiento.

El concepto de participación ciudadana se ha abordado de formas muy diversas. Esto ha generado un carácter polisémico del concepto que ha dificultado una noción general del mismo; sin embargo, ha permitido un enriquecimiento de nociones que se le vinculan en su análisis. En términos generales, podemos entender a la participación ciudadana como el proceso de intervención e injerencia de los individuos en los asuntos públicos, que tiene como principio la propia identificación de los individuos como ciudadanos al formar parte de una comunidad social y política específica. Por lo tanto, es el proceso social resultante de la acción intencionada tanto de individuos como de grupos que persiguen una meta específica, tomando en consideración sus intereses y las relaciones sociales y de poder con las que cuenten (Velásquez y González, 2003).

La participación ciudadana puede variar de acuerdo con el tipo de gobierno, su grado de democracia, las instituciones, la capacidad organizativa de la sociedad y, en general, con las relaciones entre la propia sociedad con el gobierno. En este sentido, hay autores que destacan la importancia de esta relación sociedad-Estado, entendiéndola como los procesos de interacción, comunicación e incluso de diferenciación que se dan entre ellos (Espinosa, 2004). Asimismo, estas diferentes formas de relación también influyen en el tipo de democracia y en que ésta se consolide o bien fracase a largo plazo, por lo tanto también podemos destacar el vínculo permanente que existe entre los conceptos de democracia y participación ciudadana, pues como menciona O’Donnell:

como una forma política eficaz en un territorio determinado, la democracia está necesariamente vinculada a la ciudadanía, y ésta sólo puede existir dentro de la legalidad de un Estado democrático. La universalización total de la ciudadanía es un ideal al que se acercan, en mayor o menor grado de proximidad, las democracias realmente existentes. (1993: 74)

Los cambios de gobierno que en la región y en nuestro país apuntaron a formas más democráticas de participación permitieron la generación de nuevas formas asociativas y de vinculación de los individuos, que redundaron a su vez en un renovado uso de los espacios públicos, lo cual dio cuenta, de forma empírica, de que la consolidación democrática depende en gran medida de la participación ciudadana. Sin embargo, y una vez superado el hecho de que los ciudadanos puedan ejercer de forma libre sus derechos políticos, es necesario que participen de forma más activa en otros espacios y ámbitos de las tareas públicas (Prats y Catalá, 1996; Giddens, 2000; Fleury, 2004), lo que 
generaría, a su vez, un orden institucional diferente que fuera abierto y plural (Calderón, 1995).

Ahora bien, la participación ciudadana puede darse principalmente de dos formas: institucionalizada o autónoma. En su forma institucionalizada, la participación se da dentro de los estatutos reglamentarios del marco normativo y legal y puede dar como resultado una mayor legitimidad del gobierno, una promoción de la cultura democrática, así como una más eficaz forma de toma de decisiones y de una mejor gestión pública. La autónoma, por su parte, se genera desde la propia sociedad civil sin tomar en consideración a las instituciones del gobierno (Ziccardi, 1998).

Por otro lado, la participación puede configurarse como administrativa o política. La participación administrativa es despolitizada, pues no conlleva una redistribución del poder social, sino una racionalidad meramente instrumental que toma en consideración a los beneficiarios que están involucrados de forma directa en la participación; la participación ciudadana política tiene por objetivo intervenir de cierta forma en los temas y en los balances de poder social más amplios. Este último tipo de participación ciudadana, que es la que más interesa en este trabajo, requiere de una mayor información, pues se da mediante la utilización y manifestación de argumentos racionales (Cunill, 2008).

En este punto, surge la necesidad de comprender el concepto de participación ciudadana y el del papel que desempeña en la denominada era de la información y posterior sociedad del conocimiento. ${ }^{5}$

El surgimiento de "la era de la información" puede ser identificado a la par que la revolución en el campo de las nuevas tecnologías de la información ${ }^{6}$ y comunicación que impactaron el funcionamiento económico, social y cultural tanto de las naciones como de sus interacciones entre ellas (Castells, 1999). En este sentido, y haciendo una diferenciación entre el desarrollo basado en el tema informacional con los desarrollos agrario e industrial, Castells menciona que "en el nuevo modo de desarrollo informacional, la fuente de la productividad estriba en la tecnología de la generación del conocimiento, el procesamiento de la información y la comunicación de símbolos" (1999: 42-43). Por lo tanto, en esta era de la información nos podemos encontrar con que

5 En este orden de ideas, Clark (1997) establece que la sociedad del conocimiento se podría considerar como una etapa posterior de la civilización a la llamada era de la información.

6 Para Castells (1999: 32) estas nuevas tecnologías están relacionadas con "el conjunto convergente de tecnologías de la microelectrónica, la informática (máquinas y software), las telecomunicaciones/televisión/radio y la optoelectrónica" además de la inclusión de "la ingeniería genética y su conjunto de desarrollos y aplicaciones en expansión". 
[...] los indicadores del crecimiento tecnológico en el sector informático y de comunicaciones, su impacto sobre las estructuras socionacionales y la profundización de la densidad de las relaciones sociales que de ellos se derivan nos muestran que el escenario de un futuro tal vez no tan predecible hace unas décadas -en relación con los desarrollos posibles- ya está aquí. (Bernal-Meza y Masera, 2007: 92)

Por lo anterior, podemos relacionar a la era de la información con un desarrollo que impacta directamente a la sociedad; dicho desarrollo amplía considerablemente la potencialidad de los individuos para obtener, generar y compartir información de forma instantánea, prácticamente desde cualquier ubicación y de muy diversas formas. Sin embargo, se debe tener en consideración que con el uso de este concepto se "hace referencia a un nuevo paradigma, cuyos criterios orientadores dan cuenta del camino hacia un tipo de sociedad emergente y en construcción, resultante de la acción de los sistemas tecnológicos y de los progresivos procesos de digitalización" (Bernal-Meza y Masera, 2007: 94). Ese tipo de sociedad emergente es denominada por muchos autores como la sociedad del conocimiento.

Sakaiya (1995) fue quien de cierta forma extendió el uso del concepto de sociedad del conocimiento al mencionar que, dejando de lado únicamente la satisfacción de cuestiones materiales, las sociedades darían más importancia a las cuestiones inmateriales, como el propio conocimiento, y que justamente ese conocimiento se constituiría como posible motor del desarrollo de las naciones. Esto de cierta forma fue un rompimiento en cuanto a las tendencias que apuntalaban al desarrollo vinculándolo con las sociedades industrializadas y con el aumento de capital.

Drucker (1974) fue uno de los primeros autores en referirse al término sociedad del conocimiento, argumentando precisamente que el poder de la economía estaba dejando de ser el resultado de la utilización del capital financiero o los recursos naturales, y que a su vez estos elementos daban paso al conocimiento como el componente esencial para el crecimiento económico. Incluso Drucker llegó a postular que los nuevos líderes sociales serían aquéllos con sólidos conocimientos o "profesionales del conocimiento", dejando en un segundo término a los que sólo tenían recursos financieros. Otra corriente (Hansson, 2002) apunta a señalar que vivimos ya en una sociedad del conocimiento, marcada por el desarrollo y evolución tecnológica que ha traído como resultado un considerable aumento en cuanto a la cantidad de conocimientos; y, más importante aún, coinciden en que aspectos como la riqueza, el bienestar e incluso la felicidad de las personas ya no sólo pueden ser medidos en términos productivos y de mercancías, sino en cuanto al nivel y calidad de los conocimientos de los individuos. 
Cabe subrayar que en la sociedad del conocimiento se destaca que con fenómenos como el de la globalización, las nuevas tecnologías de la información y el propio conocimiento, se puede alcanzar un correcto funcionamiento de las empresas y la actividad económica en general, así como mejorar las relaciones y la interacción entre los ciudadanos y entre éstos con las organizaciones públicas. Con esta valorización del conocimiento, el papel del ciudadano puede destacar como principal activo competitivo, dejando de lado su análisis exclusivo como recursos humanos.

En este punto es pertinente señalar que los conceptos de sociedad del conocimiento y era de la información han llegado a ser utilizados indistintamente como si fueran sinónimos e inclusive como si se refirieran a lo mismo; sin embargo, cada uno de ellos hace referencia y pone hincapié en aspectos específicos de análisis. Mientras que en la era de la información se hace referencia al gran aumento en cuanto a la disponibilidad de información por medio del uso de las tecnologías de la información y comunicación, como es el acceso a Internet, en la sociedad del conocimiento se pone énfasis en el elemento del conocimiento de los individuos como factor de desarrollo y riqueza; es decir, mientras que una hace referencia a la enorme disponibilidad de datos e información, la otra remite a la verdadera utilización y asimilación de dicha información por los individuos. Esta diferenciación no resulta menor, pues como señala Ríos (2014: 148) el "cambio de percepción de la información al conocimiento es vital para comprender el cambio de paradigma de desarrollo social", por lo tanto no debe confundirse el uso de estos conceptos ya que "el nacimiento de la Sociedad de la información basada en la revolución de las nuevas tecnologías es tan sólo un instrumento para alcanzar el modelo de sociedad del conocimiento" (Ríos, 2014: 149).

Las discusiones sobre los conceptos de la era de la información y sociedad del conocimiento resultan muy útiles al reflexionar en torno al concepto de ciudadanía y en específico al de participación ciudadana, pues los análisis apuntan a reconocer a la información y al conocimiento como variables principales, incluso sobre los recursos naturales y económicos, en la generación y distribución de poder en las sociedades modernas, lo cual impacta la dinámica interna de la propia sociedad (Webster, 1995; Thurow, 1996). Toffler (1990) menciona además que, a diferencia de los recursos naturales y económicos, el conocimiento no se gasta, sino que tiende a ampliarse mediante la generación de más conocimiento. Sin embargo, como se ha señalado anteriormente en este documento, para la correcta generación de conocimiento e información se torna fundamental un ambiente democrático en donde prime la libertad de ideas y la posibilidad de allegarse información útil para los ciudadanos que la necesitan. En este escenario, quedaría en entredicho el 
optimismo de Toffler (1990) cuando argumenta sobre la democratización de la distribución del conocimiento, mencionando que aun el pobre y el débil fácilmente la pueden adquirir, pues se tienen que considerar los problemas de acceso, así como el precio de la información y desmitificar la noción de que ésta no cuesta (Morales, 1990).

Una vez revisados los conceptos de información, ciudadanía, era de la información y sociedad del conocimiento, surge la necesidad de analizar la problemática relacionada con los bajos niveles de participación ciudadana en la gestión de políticas públicas imperante en mayor o menor grado en los países de la región. Este problema se complejiza al tomar en consideración que las circunstancias analizadas pueden actuar de manera recíproca como causa y efecto; es decir, por un lado los ciudadanos necesitan de mayor información para argumentar de mejor forma sus necesidades e ideas para que impacten de forma positiva su participación en los asuntos públicos y en la elaboración de las políticas públicas; pero por otro lado, se necesitan mejores políticas públicas para que los ciudadanos tengan los canales necesarios para allegarse la información y, con ello, cuenten con mejores elementos para participar. La salida al dialelo aquí propuesto está encaminada a la adopción de un modelo de gobernanza en la gestión de las políticas públicas, modelo que será analizado en el siguiente y último apartado.

\section{EL MODELO DE LA GOBERNANZA EN LA GESTIÓN DE POLÍTICAS PÚBLICAS}

Se ha mencionado que la "política pública está hecha de palabras" (Majone, 1997: 2); en este sentido, en cualquiera de sus modalidades, oral o escrita, la argumentación resulta necesaria en el proceso de elaboración y gestión de las políticas, máxime en el contexto de los gobiernos democráticos, caracterizados por la posibilidad de que los diferentes actores interactúen mediante la discusión y el debate de las ideas que conlleven a acuerdos y acciones concretas.

En un modelo idealizado en la gestión de las políticas llevadas a cabo en un Estado democrático se daría un proceso de expresión de ideas, necesidades y preocupaciones sobre temas comunes; estos elementos serían retomados por los candidatos a ocupar cargos de representación popular, quienes tendrían que saber plasmar en planes, proyectos y programas las necesidades recogidas en el proceso de expresión de ideas y demandas, de forma que los electores pudieran decidir, mediante las elecciones, qué candidato se acerca más a satisfacer las necesidades y problemas que los aquejan. Posteriormente el poder legislativo traduciría los proyectos en leyes, para al final convertirse en políticas públicas por parte del ejecutivo (Majone, 1997). 
Este supuesto ideal está lejos de la realidad, pues existe un desigual acceso y distribución de la información que propicia el conocimiento y la participación mediante la argumentación. Esto se ve reflejado en un problema de dos vertientes: por un lado surgen grupos que dominan y concentran mayor cantidad y calidad de información, los cuales tienden a ejercer poder e influencia en la gestión de políticas y en la forma de tratar los asuntos públicos; y por el otro, se generan bajos niveles de participación ciudadana e involucramiento de un amplio sector de la sociedad en los asuntos públicos. Lo anterior da muestras de que existe una insatisfacción de las necesidades de información, lo que frena a su vez "la ampliación de los conocimientos de los individuos y [...] un desarrollo intelectual que incide en la formación de las personas con miras a lograr un mejor escenario de vida" (Ramírez, 2013: $\mathrm{XV}$ ). Por ello, surge la necesidad de pensar y reflexionar en nuevas formas de gobierno que permitan crear mecanismos que propicien una mejor distribución de información y con ello una participación más amplia en los asuntos públicos.

En este sentido resultan interesantes los aportes del modelo de gobernan$z a$, que difieren del de gobernabilidad, y aunque no es objeto de este trabajo entrar en la discusión minuciosa de las diferencias entre estos modelos, es oportuno, en un primer momento, desarrollar una breve sistematización de las perspectivas analíticas que han abordado su estudio, con objeto de tener un mejor entendimiento del concepto de gobernanza.

En primera instancia el concepto de gobernabilidad empieza a ser utilizado como un planteamiento que tomaba en consideración la necesidad de superar tanto los desajustes ocasionados por las demandas y exigencias sociales como la capacidad gubernamental de atender éstas por medio de su puesta en consideración en las agendas públicas. Lo anterior fue resultado de la situación que caracterizaba al aparato gubernamental de muchos países en la década de los años setenta (Crozier, Hungtinton y Watanuki, 1975). Asimismo, los principios de gobernabilidad implicaban la necesidad de atender la crisis relacionada con el ámbito financiero, así como la de legitimidad, en los términos de eficiencia y eficacia en los que se encontraba el sector público.

Ante la cada vez más notoria crisis por la que atravesaban los Estados de Bienestar, el término hizo referencia a la capacidad de ajuste de los gobiernos para atender los nuevos retos y exigencias sociales que se les presentaban. De lo anterior se desprende que a grandes rasgos se pueda definir a la gobernabilidad como la capacidad gubernamental y de gestión pública. De igual forma, habría que sumar que con esa buena forma de gobernar, aunada a una mejora en la situación política por la expansión de los derechos y oportunidades de los ciudadanos, se podría evitar la posibilidad de retroceder a go- 
biernos autoritarios, lo que agrega un aspecto relacionado con la estabilidad política mediante la consolidación o el afianzamiento democrático (O'Donnell, 1979; Przeworski y Wallerstein, 1988).

Sin embargo, el concepto de gobernabilidad se ha definido en los últimos tiempos más por su contraparte o aspecto negativo, la ingobernabilidad (Coppedge, 1996), debido a aspectos como la sobrecarga económica del Estado, el aumento de demandas públicas no atendidas y aun por problemas de racionalidad entre los intereses del mercado y el crecimiento de la esfera de los servicios sociales (Pasquino, 2005; Mayntz, 2000). En este sentido, hay autores que han mencionado que la gobernabilidad es sólo una ideología conservadora de la crisis (Offe, 1979).

Por otro lado, el concepto de la gobernanza (Aguilar, 2006; Kauffman, Kraay y Zoido-Labaton, 2000; Peters, 1998; Brugué, Gomà y Subirats, 2005; Torres y Ramos, 2008, 2012; Mayntz, 2000, 2002; Scharpf, 2000, 2001; Camou, 2000; entre otros) ha sido utilizado desde múltiples enfoques, la mayoría de ellos convergen en definirlo como una forma de llevar a cabo la ejecución de los procesos, así como una forma más plural de tomar las decisiones públicas mediante la delegación de poder a los actores involucrados en el tema en cuestión. De igual forma, se ha utilizado para dar cuenta del establecimiento de una gestión público-privada (mixta) para alcanzar acuerdos de forma más horizontal, así como consensos que conlleven a una disminución de las diferencias jerárquicas y la eliminación de la visión del Estado como único capacitado para tomar decisiones públicas (Torres y Ramos, 2008).

Mayntz (2000) atribuye dos elementos novedosos en la definición de gobernanza, el primero entendiéndola como "un nuevo estilo de gobernar, distinto del modelo de control jerárquico y caracterizado por un mayor grado de cooperación y por la interacción entre el Estado y los actores no estatales al interior de redes decisionales mixtas entre lo público y lo privado" (Mayntz, 2000: 1), mientras que el segundo elemento está relacionado con caracterizar a la gobernanza como "una modalidad distinta de coordinación de las acciones individuales, entendidas como formas primarias de construcción de orden social" (Mayntz, 2000: 1). Con esta forma de gestión más participativa, el gobierno puede trabajar más allá de sus límites convencionales mediante la utilización de redes y procesos de participación pública, con objeto de mejorar sus capacidades para anticipar, intervenir, innovar, aprender, agilizar y, finalmente, recuperase de las crisis (Bourgon, 2010).

Existe una vertiente epistemológica sobre el concepto de gobernanza que se considera pertinente destacar, la cual surge a partir de los movimientos y reflexiones críticas que se desarrollaron en las décadas de los setenta y ochenta, en donde el concepto fue concebido como una dirección descen- 
tralizada en redes horizontales de organizaciones mixtas público-privadas. ${ }^{7}$ Desde esta misma vertiente, el concepto fue considerado como la construcción de un consenso horizontal crítico de las jerarquías. Lo anterior permitió el entendimiento de las instituciones como redes de decisiones horizontales, las cuales cuestionaron la funcionalidad de instituciones y organismos de corte centralizado.

A partir de los años noventa se fue configurando un paradigma con relación a la posmodernidad de la vida política y la descentralización. Una vertiente de este paradigma pretendió dar cuenta de los beneficios que los lazos sociales podían dar a la capacidad de gobierno mediante un "empoderamiento" a la participación social, por medio de la implementación de redes de políticas públicas y una cultura de la rendición de cuentas o accountability (Peters, 1998). Esto apuntó a una reestructuración relacionada con las funciones tradicionalmente desempeñadas por el sistema político-administrativo del Estado, pasando así de un control único de los procesos políticos a una cada vez mayor negociación con los actores públicos y privados. Algunos autores sugieren que con este proceso se difuminó, cada vez más, la frontera entro lo público y lo privado (Börzel, 1998; Kenis y Schneider, 1991; Kohler-Koch, 1996).

En este punto resulta oportuno señalar las dimensiones de la gobernanza, las cuales son:

- Construcción desde abajo hacia arriba de procesos de coordinación de las organizaciones e instituciones de los tres niveles de gobierno.

- Gestión y esclarecimiento de las propuestas de los interesados para transformarlas en política pública.

- Acuerdos público-privados y sociales con indicadores de evaluación de desempeño.

- Dirección descentralizada de la gestión.

- Cultura de la transparencia y rendición de cuentas.

- Disminución de los costos de transacción que pueden medirse por medio de la densidad de las relaciones de confianza y reciprocidad, para facilitar la difusión de innovaciones en una escala espacial dada.

El principal rasgo de la gobernanza que es preciso enfatizar, con respecto a la utilidad del análisis de la participación ciudadana en la gestión de políti-

7 Se ha utilizado el término partenariado para referirse al desarrollo basado en una participación amplia de diferentes actores que, mediante el diálogo y el discurso, logran llegar a acuerdos conjuntos que permiten impactar la formulación de políticas, programas y acciones; es decir, los receptores de políticas se convierten en actores importantes para definir las acciones gubernamentales. 
cas públicas, es aquel donde se aborda el concepto desde el punto de vista de la coordinación y articulación de los actores en espacios geográficos, social y culturalmente determinados; es decir, en lugares y territorios bien definidos. Para dar ejemplo de ello, en el caso de una mejora en cuanto a las políticas de información, será necesario involucrar y "coordinar" a actores como agencias internacionales, tomadores de decisiones públicas (nacionales, estatales y municipales), organizaciones no gubernamentales, generadores de información, difusores y proveedores, usuarios, responsables de medios, personal de bibliotecas, especialistas en bibliotecología y ciencias afines a la información y demás individuos involucrados en el tema. En este sentido, se ha mencionado que con la gobernanza se

permite la construcción y consolidación de redes, intercambios solidarios y asociaciones, es decir, de capital social, pero a su vez, es un producto de los intercambios y las reglas construidas desde abajo 'bottom up' y de la coordinación con las instituciones desde arriba 'top down' lo que a su vez, permite un aumento de las capacidades (positivas) de los ciudadanos. (Torres y Ramos, 2012: 104)

De igual forma, la utilización de un modelo de gobernanza en la gestión de políticas públicas implicaría la puesta en marcha de un sistema de reglas, que pueden ser tanto formales como informales, que tiendan a marcar las pautas de interacción e intercambios en la esfera pública, y que también incluyan la participación plural de actores del ámbito de los poderes públicos, sociales y económicos; con lo cual, y mediante la utilización de mecanismos de rendición de cuentas, se logre tanto una mayor legitimidad de las acciones gubernamentales (Aguilar, 2006; Pierre y Peters, 2000; Williamson, 1979, 1994; Mayntz, 2002; McCarney, Halfani y Rodríguez, 1998) como una mejora en la disponibilidad y acceso a información relevante para que los ciudadanos cuenten con mejores elementos para fundamentar sus discursos y su aparición en la esfera pública.

\section{ReFLEXIONES FINALES}

Uno de los objetivos de este trabajo fue abordar los cambios que ha tenido el concepto de ciudadanía, de ser un concepto que sólo atribuía derechos a los individuos a uno polisémico que incluye en la actualidad una visión más participativa de la actividad y vinculación de los individuos en los asuntos públicos. Este cambio está relacionado, a su vez, con las transformaciones democráticas, en donde se ha argumentado que para mejorar los niveles democráticos es necesario pasar de una democracia representativa, caracteriza- 
da principalmente por la capacidad de elección de representantes públicos, a una democracia activa, en la cual los ciudadanos participen constantemente en los asuntos públicos.

Sin embargo, para que esta participación esté bien fundamentada, resulta necesaria la información, con objeto de lograr una buena aparición en la esfera pública, caracterizada por la calidad de la argumentación y por la claridad de transmisión de ideas. En este sentido, se destacó la importancia de la información como elemento esencial en la conformación de una ciudadanía más participativa. No obstante, en la región latinoamericana aún persisten serias desigualdades en cuanto al acceso y el uso de la información, lo que redunda a su vez en una diferenciación entre la calidad de participación. Lo anterior genera que persistan élites que concentran un gran cúmulo de información, lo que impacta favorablemente en su participación y la inclusión de sus necesidades en la agenda pública, y un amplio sector desinformado, que no participa y que desconoce gran parte de los derechos que podrían darle un mejor modo de vida.

Ante esta situación, se propuso el modelo de gestión de la gobernanza, reconociendo la complejidad de los problemas relacionados con la distribución y acceso a la información, pues con este modelo se toma en consideración una participación más plural y un modo de toma de decisiones más cooperativo. En este escenario, el gobierno deja de ser el único actor facultado para la toma de decisiones y se destacan sus atributos como coordinador de los actores interesados en la participación de los asuntos y gestión de políticas públicas.

Por lo tanto, la gobernanza, desde el punto de vista que se ha abordado, constituye un elemento de integración de los ciudadanos con las actividades del Estado, en particular con la gestión de políticas públicas, que puede generar sinergias positivas, un desarrollo en las capacidades de participación y la posibilidad de un mejoramiento en los canales de acceso, distribución y generación de información. En este mismo sentido, con la gobernanza destaca la posibilidad de que fluya más la información y el conocimiento, al tratarse de un modelo más horizontal de gobierno y de toma de decisiones públicas, donde los diferentes actores formen parte tanto en la toma de decisiones como en la obtención de resultados, que deriven en un proceso más transparente de gestión pública.

Sin embargo, a la par de los resultados del trabajo, se abren una serie de cuestionamientos que pueden dar lugar a nuevos estudios que ayuden a enriquecer el debate de las temáticas abordadas, tales como ¿cuáles son los cambios que han existido en el plano legislativo relacionados con el acceso a la información pública y cómo han impactado éstos la participación ciu- 
dadana?, ¿cuál es el papel de las bibliotecas públicas en la formación de ciudadanos?, ¿existen elementos de gobernanza en ámbitos más reducidos de análisis, como son el ámbito estatal o municipal?, y finalmente ¿qué otras iniciativas, tanto gubernamentales como sociales, pueden impactar en la formación de un modelo más cercano al de gobernanza?

\section{Bibliografía}

Aguilar, L. (2006), Gobernanza y gestión pública, México, Fondo de Cultura Económica.

Aguirre, R. (2003), Género, Ciudadania social y trabajo, Montevideo, Universidad de la República.

Arendt, H. (1993), La condición bumana, Barcelona, Paidós.

Bernal-Meza, R. y Masera, G. (2007), "Sociedad de la información: etapa posterior de la globalización/mundalización. Desafíos y riesgos para América Latina”, en Realidad Económica, núm. 227, abril-mayo, pp. 90-116.

Bolos, S. (2008) (coord.), Mujeres y Espacio Público: construcción y ejercicio de la ciudadanía, México, Universidad Iberoamericana, Instituto Nacional de las Mujeres.

Borja, J. (1998), "Ciudadanía y espacio público", en Revista Ambiente y Desarrollo, PUJ, Bogotá, vol. XIV, núm. 3, pp. 13-22.

Börzel, T. (1998), "Le reti di attori pubblici e privati nella regolazione europea”, en Stato e Mercato, núm. 54, pp. 389-431.

Bourgon, J. (2010), "Propósito público, autoridad gubernamental y poder colectivo", en Revista del CLAD Reforma y Democracia, núm. 46, pp. 1-23.

Brugué, Q.; Gomà, R. y Subirats, J. (2005), "Gobernar ciudades y territorios en la sociedad de redes", en Revista Reforma y Democracia, Centro Latinoamericano de Administración para el Desarrollo (CLAD), núm. 32, junio, pp. 5-18.

Calderón, F. (1995), Sociedad sin atajos, Buenos Aires, Paidós.

Camou, A. (2000), "La múltiple (in) gobernabilidad: elementos para un análisis conceptual”, en Revista Mexicana de Sociología, vol. 62, núm. 4, octubre-diciembre, pp. 159-188.

Canto, M. (2008), "Participación ciudadana: la gobernanza de las sociedades complejas”, en Revista Política y Cultura, núm. 30, pp. 1-25.

Capron, G. y Monnet, J. (2003), "Una retórica progresista para un urbanismo conservador: la protección de los centros históricos en América Latina”, en P. Ramírez (coord.), Espacio público y reconstrucción de ciudadanía, México, Miguel Ángel Porrúa y FLACSO, pp. 105-136.

Castells, M. (1999), La era de la información. Economía sociedad y cultura, Vol. I La sociedad red, México, Siglo XXI Editores. (2001), La Galaxia Internet, Barcelona, Plaza \& Janés. 
Clark, I. (1997), Acerca de la información como fetiche, ¿Sociedad del conocimiento? Disponible en: http://www.parquechasweb.com. ar/parquechas/notas/Nota_soconocimiento270607.htm [Fecha de consulta: 13 de febrero de 2013].

Coppedge, M. (1996), "El concepto de gobernabilidad. Modelos positivos y negativos", en PNUD CORDES (comp.), Ecuador: un problema de gobernabilidad, Quito, PNUD CORDES.

Craston, M. (1983), "Are There Any Human Rights?", en Journal of the American Academy of Arts and Sciences, vol. 112, núm. 4, pp. 1-17.

Crozier, M.; Hungtinton, S. y Watanuki, J. (1975), The Crises of Democracy, Report on the Governability of Democracies to the Trilateral Commission, Nueva York, New York University Press.

Cunill, N. (2008), "La construcción de ciudadanía desde una institucionalidad pública amplia”, en R. Mariani (coord.), Contribuciones al debate: Democracia/Estado/Ciudadanía. Hacia un Estado de y para la democracia en América Latina, vol. II, PNUD-UE, pp. 113-138.

Drucker, P. (1974), Management tasks, responsibilities, practices, Nueva York, Harper Collins Publishers.

Espinosa, M. (2004), "Historia y cultura política de la participación ciudadana en la Ciudad de México: entre los condicionamientos del sistema y el ensueño cívico", en Andamios, núm. 1, pp. 9-50.

Fleury, S. (2004), "Construcción de ciudadanía en entornos de desigualdad”, en C. Binetti y F. Carrillo (eds.), ¿Democracia con desigualdad? Una mirada de Europa hacia América Latina, Nueva York, Banco Interamericano de Desarrollo, pp. 133-169.

García, N. (1995), Consumidores y ciudadanos, México, Grijalbo.

Giddens, A. (2000), Un mundo desbocado, los efectos de la globalización en nuestras vidas, Madrid, Taurus.

Giglia, A. y Winocur, R. (1996), "La participación en la radio: entre inquietudes ciudadanas y estrategias mediáticas”, en Perfiles Latinoamericanos, año 5, núm. 9, pp. 73-84.

Guerrero, M. (2006), "Medios de comunicación y la función de transparencia”, en Cuadernos de Transparencia, núm. 11, México, Instituto Federal de Acceso a la Información y Protección de Datos.

Habermas, J. (1987), Teoría de la acción comunicativa. Volumen 2: Crítica de la razón funcionalista, Madrid, Taurus.

Hansson, S. (2002), "Las inseguridades en la sociedad del conocimiento”, en Revista Internacional de Ciencias Sociales, núm. 171, pp. 112. Disponible en: http://www.oei.es/salactsi/rics171.htm [Fecha de consulta: 21 de abril de 2014].

Hoffman, D.; Novak, T. y Schlosser, A. (2001), "The evolution of the digital divide: Examining the relationship of race to internet access and usage over time", en B. Compaine (coord.) The Digital Divide: Facing a Crisis or Creating a Myth?, Cambridge, Massachussets, The MIT Press, pp. 47-97. 
Kauffman, D.; Kraay, A. y Zoido-Labaton, P. (2000), “Aggregating Governance Indicators”, en Policy Research Working Paper, núm. 2195, pp. 1-35. Disponible en: http://www.worldbank.org/wbi/go vernance/pdf/agg_ind.pdf [Fecha de consulta: 25 de mayo de 2008].

Kenis, K. y Schneider, V. (1991), "Policy Networks and Policy Analysis”, en B. Marin y R. Mayntz (eds.), Policy Networks: Empirical Evidence and Theoretical Considerations, Frankfurt, Campus Verlag, pp. 25-59.

Kohler-Koch, B. (1996), "Catching up with change: the transformation of governance in the European Union", en Journal of European Public Policy, núm. 3, pp. 359-380.

Lechner, N. (2000), “Nuevas Ciudadanías”, en Revista de Estudios Sociales, núm. 5, enero, pp. 25-31.

Majone, G. (1997), Evidencia, argumentación y persuasión en la formulación de políticas, México, Fondo de Cultura Económica.

Martín-Barbero, J. (1999), "El miedo a los medios. Política, comunicación y nuevos modos de representación”, en Nueva Sociedad, núm. 161, mayo-junio, pp. 43-56.

- (2001), Claves de Debate: Televisión pública, televisión cultural: entre la renovación y la invención, Guadalajara, ITESO.

Marshall, T. H. (1997), "Ciudadanía y Clase Social”, en Revista Española de Investigaciones Sociológicas, núm. 79, pp. 279-334.

Mayntz, R. (2000), "Nuevos desafíos de la teoría de Governance", en Instituciones y desarrollo, núm. 07, pp. 1-10. Disponible en: http:// www.iigov.org/id/index.drt?edi=187626 [Fecha de consulta: 15 de enero de 2014].

_ (2002), "National states and global governance", trabajo presentado en VII Congreso Internacional del CLAD sobre la Reforma del estado y de la Administración Pública, 8-11 de octubre, Lisboa.

McCarney, P.; Halfani, M. y Rodríguez, A. (1998), "Hacia una definición de 'gobernanza”, en Proposiciones, núm. 28, pp. 118-155.

Molyneux, M. (2000), "Gender and Citizenship in Comparative Perspective”, en J. Cook, J. Roberts y G. Waylen (eds.), Towards a Gendered Political Economy, Houndsmills, Macmillan, pp. 121-144.

Morales, E. (1990), Sociedad e información, trabajo presentado en la 9 $9^{a}$ Reunión Interamericana de Bibliotecarios y Documentalistas Agrícolas, AIBDA/II-CA, San José.

O'Donnell, G. (1979), Democracy and Bureaucratic Authoritarianism in Latin America, Berkeley, Institute of International Studies.

— (1993), "Estado, Democratización y ciudadanía", en Nueva Sociedad, núm. 128, noviembre-diciembre, pp. 62-87.

Offe, C. (1979), Ingobernabilidad. Sobre el renacimiento de teorías conservadoras de la crisis, Frankfurt, EDUCA.

Pasquino, G. (2005), "Gobernabilidad", en N. Bobbio, I. Mateucci y G. Pasquino (coords.), Diccionario de Política, 14 ed. en español, México, Siglo XXI Editores, pp. 703-710. 
Peña, C. (2005), “Ciudadanía y reconocimiento: el lugar de las minorías”, en Revista Derecho y Humanidades, núm. 11, pp. 99-103.

Peters, G. (1998), R. A. W. Rhodes, Understanding Governance: Policy Networks, Governance, Reflexivity and Accountability, Blackwell Publishing, Public Administration, vol. 76, núm. 2, pp. 394-396.

Pierre, J. y Peters, G. (2000), Governance, politics and the state, Nueva York, Macmillan.

Prats y Catalá, J. (1996), Gobernabilidad democrática en América Latina finisecular, Washington, BID.

Programa de las Naciones Unidas para el Desarrollo (2003), El acceso a la Información, nota práctica, Nueva York, PNUD.

Przeworski, A. y Wallerstein, M. (1988), "Structural Dependence of the State on Capital”, en American Political Science Review, vol. 82, núm. 1, marzo, pp. 11-29.

Ramírez, C. (2013), La satisfacción de las necesidades de información y el cambio de identidad en la comunidad indígena amuzga, México, UNAM-IIBI.

Ramírez, P. (2003), "El espacio público: ciudad y ciudadanía. De los conceptos a los problemas de la vida pública local”, en P. Ramírez (coord.), Espacio público y reconstrucción de ciudadanía, México, FLACSO, Miguel Ángel Porrúa, pp. 31-58.

Ríos, J. (2014), "El concepto de información: dimensiones bibliotecológica, sociológica y cognoscitiva”, en Investigación Bibliotecológica, vol. 28, núm. 62, pp. 143-179.

Roche, M. (1987), "Citizenship, social theory, and social change”, en Theory and Society, vol. 16, núm. 3, pp. 363-399.

Rodríguez, A. (2006), La brecha digital y sus determinantes, México, UNAM-CUIB.

Sakaiya, T. (1995), Historia del Futuro. La sociedad del conocimiento, Santiago de Chile, Andrés Bello.

Scharpf, F. (2000), "Economic Changes, Vulnerabilities, and Institutional Capabilities”, en F. Scharpf y V. Schmidt (eds.), Welfare and Work in the Open Economy Volume I: From Vulnerability to Competitiveness in Comparative Perspective, Oxford, Oxford Scholarship, pp. 21-124.

- (2001), European Governance: Common Concerns vs. The Challenge of Diversity, MPIfG Working Paper 01/6. Disponible en: http://www.mpi-fg-koeln.mpg.de/pu/workpap/wp01-6.htm [Fecha de consulta: 16 de diciembre de 2013].

Servon, L. (2002), Bridging the Digital Divide. Technology, community and public policy, Oxford, Blackwell Publishing.

Thurow, L. (1996), El futuro del capitalismo, Buenos Aires, Javier Vergara Editor.

Toffler, A. (1990), El cambio del poder, Barcelona, Plaza \& Janés. 
Torres, G. y Ramos, A. (2008), "Gobernanza y territorios. Notas para la implementación de políticas para el desarrollo”, en Revista Mexicana de Ciencias Politicas y Sociales, vol. 50, núm. 203, mayo-agosto, pp. 75-95.

—_ y Ramos G. (2012), "Nueva gestión de lo local. Desarrollo rural y construcción de sistemas agroalimentarios”, en B. Lerner, R. Uvalle y R. Moreno (coords.), Gobernabilidad y gobernanza en los albores del Siglo XXI y Reflexiones sobre el México contemporáneo, Estado de México, IIS-UNAM, IAPEM, pp. 99-136.

Turner, B. (1990), "Outline of a Theory of Citizenship", en The Journal of the British Sociological Association, vol. 24, núm. 2, pp.189217.

Velásquez, F. y González, E. (2003), ¿Qué ha pasado con la participación ciudadana en Colombia?, Bogotá, Fundación Corona.

Vieira, L. (1998), “Ciudadanía y Control Social”, en L. Bresser-Pereira y N. Cunill (eds.), Lo Público No-Estatal en la Reforma del Estado, Buenos Aires, Paidós.

Webster, F. (1995), Theories of the Information Society, Londres, Routledge.

Williamson, O. (1979), "Transaction-Cost Economics: The Governance of Contractual Relations", en Journal of Law and Economics, vol. 22, núm. 2, octubre, pp. 233-261.

(1994), The Mechanisms of Governance, Nueva York, Oxford University Press.

Winocur, R. (2002), Ciudadanos en los medios. La construcción de lo público en la radio, Buenos Aires, Gedisa.

— (2003), "La invención mediática de la ciudadanía”, en P. Ramírez (coord.), Espacio público y reconstrucción de ciudadanía, México, Miguel Ángel Porrúa, FLACSO, pp. 231-277.

Ziccardi, A. (1998), Gobernabilidad y participación ciudadana en la ciudad capital, México, UNAM, Miguel Ángel Porrúa. 